\author{
Ecología
}

\title{
Diversidad de briófitos en fragmentos de bosque seco tropical, Montes de María, Sucre, Colombia
}

\author{
Bryophyte diversity in a tropical dry forest fragment, Montes de María, Sucre, Colombia \\ Stevens García-Martínez* y Jorge D. Mercado-Gómez \\ Departamento de Biología y Química, Grupo Evolución y Sistemática Tropical, Universidad de Sucre, Cra 28 \# 5-267, Sincelejo, Colombia \\ Recibido el 16 de febrero de 2017; aceptado el 14 de junio de 2017 \\ Disponible en Internet el 6 de diciembre de 2017
}

\begin{abstract}
Resumen
En los bosques secos tropicales del Caribe colombiano la diversidad de briófitos es muy poco conocida, a pesar de ser considerado como uno de los fragmentos de bosque en mejor estado de conservación. En el presente estudio se analizó la diversidad de este grupo de plantas en los Montes de María, Sucre, Colombia. En 6 localidades fueron identificados y cuantificados los briófitos, con base en esta información se estimó su diversidad biológica $(\alpha$ y $\beta)$ y taxonómica $\left(\Delta^{+}\right.$y $\left.\Lambda^{+}\right)$. Se registraron un total de 1,125 vástagos pertenecientes a 63 especies, 42 géneros y 23 familias. Las familias con mayor riqueza fueron: Neckeraceae, Pottiaceae, Sematophyllaceae, Lejeuneaceae y Plagiochilaceae, mientras que en los géneros sobresalieron: Fissidens, Neckeropsis y Sematophyllum. Colosó y Morroa fueron las localidades más diversas y San Onofre la menos diversa, siendo afectada por la fragmentación y destrucción del bosque. Este estudio constituye el primer aporte sobre los patrones de diversidad de briófitos en esta región del país, así como sus implicaciones para conocer la estructura de los ensambles en los ecosistemas secos tropicales. (C) 2017 Universidad Nacional Autónoma de México, Instituto de Biología. Este es un artículo Open Access bajo la licencia CC BY-NC-ND (http://creativecommons.org/licenses/by-nc-nd/4.0/).
\end{abstract}

Palabras clave: Caribe; Composición; Hepáticas; Musgos; Patrones

\section{Abstract}

In the tropical dry forests of the Colombian Caribbean bryophyte diversity is little known although this area presents the best preserved forest fragments. In this study, we analyzed bryophyte diversity in the "Montes de María", Sucre, Colombia. Bryophytes were identified and quantified in 6 localities, on the basis of this information the taxonomic $\left(\Delta^{+}\right.$and $\left.\Lambda^{+}\right)$and biological $(\alpha$ and $\beta)$ diversity were calculated. A total of 1,125 individuals belonging to 63 species, 42 genera and 23 families, were registered. Species-rich families were: Neckeraceae, Pottiaceae, Sematophyllaceae, Lejeuneaceae and Plagiochilaceae; while the genera: Fissidens, Neckeropsis, Sematophyllum, Lejeunea and Plagiochila, were important. Colosó and Morroa are the most diverse localities and San Onofre has the least diversity of all, because of forest fragmentation and destruction. This study constitutes the first contribution on the diversity patterns of bryophytes of this region of the country; as well as their implications for the structure of the assemblages in dry tropical ecosystems.

(C) 2017 Universidad Nacional Autónoma de México, Instituto de Biología. This is an open access article under the CC BY-NC-ND license (http://creativecommons.org/licenses/by-nc-nd/4.0/).

Keywords: Caribbean; Composition; Liverworts; Mosses; Patterns

\section{Introducción}

El bosque seco tropical es uno de los ecosistemas más fragmentados y degradados en el Neotrópico producto del desarrollo

\footnotetext{
* Autor para correspondencia.

Correo electrónico: stgarciamtz@gmail.com (S. García-Martínez).

La revisión por pares es responsabilidad de la Universidad Nacional Autónoma de México.
}

de la ganadería y agricultura (Marulanda et al., 2003; Pizano y García, 2014; Rodríguez, Banda, Reyes y Estupiñán, 2012). En Colombia este bosque se ha visto afectado desde hace más de 50 años por actividades humanas agresivas que reducen su flora y fauna (Alvarado y Otero, 2015). Además de la perturbación antrópica, también es otra problemática de gran importancia la falta de información taxonómica sobre muchos clados. Por ejemplo, en plantas, el linaje en el que se han desarrollado gran parte de los análisis florísticos han sido las angiospermas, dejando 
de lado grupos como los pteridófitos y briófitos, de los cuales actualmente se desconoce su diversidad y distribución en los bosques estacionalmente secos del país.

En Colombia los estudios realizados a la fecha (Aguirre y Avendaño, 2008; Aguirre y Ruiz, 2001; Avendaño y Aguirre, 2007, 2009; Ruiz y Aguirre, 2003; Santos y Aguirre, 2010; Van Reenen, Griffin y Gradstein, 1984) se han enfocado en la brioflora de zonas altas (andina y paramuna), dejando a un lado las tierras bajas y valles interandinos (García, Basilio, Herazo, Mercado y Morales, 2016). En el caso del bosque seco tropical del Caribe, más específicamente en los Montes de María, área considerada como una de las zonas de bosque seco en mejor estado de conservación (Pizano y García, 2014), solo se ha registrado el análisis florístico realizado por García et al. (2016), que registraron más de 30 especies en solo 3 localidades que corresponden a $300 \mathrm{~m}^{2}$, es decir, menos del $10 \%$ de la cobertura boscosa en los Montes de María. En otras palabras, es posible que al ampliar el área de muestreo la riqueza se pueda incrementar considerablemente, con lo cual se mejoraría el estado del conocimiento sobre un grupo subvalorado en los ecosistemas estacionalmente secos de Colombia.

Por otra parte, al analizar la riqueza, composición y abundancia de especies, los estudios se han enfocado en el desarrollo y aplicación de índices que miden la entropía y no la diversidad de una comunidad (Jost, 2006). Bajo este contexto Jost (2006) propuso analizar la diversidad verdadera o real a través de varios niveles que incluyen: orden cero $\left({ }^{0} \mathrm{D}\right)$, que corresponde a la riqueza de especies $\left({ }^{0} \mathrm{D}=\mathrm{S}\right)$ y es insensible a la abundancia relativa; orden $1\left({ }^{1} \mathrm{D}\right)$, donde las especies son incluidas proporcionalmente según su abundancia en la comunidad, y por último, orden $2\left({ }^{2} \mathrm{D}\right)$ que toma en cuenta las especies dominantes o comunes. Estos niveles posibilitan la comparación de la diversidad de 2 o más áreas con condiciones ambientales diferentes (Jost, 2006), lo cual no es posible con los índices clásicos (Moreno, Barragán, Pineda y Pavón, 2011).

Asimismo, en las últimas décadas la diversidad ha sido explorada a través de diferentes fuentes de información que no incluyen necesariamente datos de riqueza y abundancia. Warwick y Clarke (1995) propusieron calcular la distinción taxonómica promedio $\left(\Delta^{+}\right)$y su variación $\left(\Lambda^{+}\right)$. Estos índices evalúan la distancia y variación taxonómica entre cada par de individuos a partir del listado de especies con todas sus categorías o niveles taxonómicos (Clarke y Warwick, 1998); es decir, permiten estimar el grado de relación taxonómica entre especies donde a mayor número de linajes distribuidos entre los niveles taxonómicos, más altos serán los valores de distinción taxonómica promedio y por ende la diversidad. Estos índices son importantes para conocer la diversidad desde otros puntos de vista y son relevantes al momento de fijar áreas prioritarias para la conservación y para el manejo de los recursos naturales (García, Moreno y Bello, 2014; Moreno et al., 2011), ya que son susceptibles a las presiones antropogénicas (Moreno et al., 2011; Warwick y Clarke, 1995).

La presente investigación tuvo como objetivo conocer la composición de briófitos y determinar si existen patrones en su diversidad a través de índices de diversidad real ( $\alpha$ y $\beta$ ), distinción y variación en la distinción taxonómica en 6 localidades que corresponden a bosques estacionalmente secos en Montes de María, departamento de Sucre, Colombia.

\section{Materiales y métodos}

Los Montes de María son considerados como una subregión del Caribe colombiano y se localizan en la parte central de los departamentos de Bolívar y Sucre, abarcando una extensión total de $6,297 \mathrm{~km}^{2}$ (Aguilera, 2013). Desde el punto de vista fisiográfico en ella se encuentra un sistema montañoso que pertenece a las estribaciones de la Serranía de San Jerónimo y se distribuye entre los municipios de Colosó, Chalán, Morroa, Ovejas, parte de Los Palmitos, San Onofre y Toluviejo (Aguilera, 2013 [fig. 1]).

Los suelos en esta área son derivados de mantos arenosos, arcillosos y de calizas del Mioceno (Halffter, 1992). Asimismo, las montañas y colinas están fuertemente escarpadas, además de presentar afloramientos rocosos y arcillolitas de origen marino (Galván, Gómez, de la Ossa y Fajardo, 2009; Promontes, 2003). Con respecto a la hidrología existe una red perteneciente a la cuenca del Golfo de Morrosquillo (Verbel, 2009) que junto a la topografía de la subregión forman un sistema de microcuencas que da origen a numerosos arroyos y cuerpos de agua de duración estacional limitada por épocas de lluvias (Aguilera, 2013).

De igual forma, en sitios como Morroa y Colosó se han registrado reservorios de aguas subterráneas. En Morroa, por ejemplo, estas aguas son captadas a través de pozos profundos de los acuíferos libres y confinados de las diferentes formaciones geológicas del área (López, 2003), así también en Colosó existen reservorios de aguas subterráneas que a la fecha no han sido estudiados en profundidad (Verbel, 2009).

Respecto al clima el régimen de lluvia es bimodal y anualmente se presentan valores desde 1,000 a $1,200 \mathrm{~mm}$ con incremento en el segundo semestre del año; la temporada de sequía está comprendida entre junio-julio y diciembre-febrero (Aguilera, 2005, 2013; Cuervo, Barbosa y de la Ossa, 1986). Finalmente, la temperatura varía entre $26-30{ }^{\circ} \mathrm{C}$ y la humedad relativa oscila entre $75 \%$ y $85 \%$ (Aguilera, 2013; Promontes, 2003).

La vegetación está conformada principalmente por bosque seco tropical, según Holdridge (1967) entre sus elementos más característicos resaltan especies de árboles como: Ampelocera edentula, Aspidosperma polyneuron, Brosimum alicastrum, Myrcia fallax y Simira cordifolia (Herazo, Mercado-Gómez, \& Mendoza, 2017). No obstante, históricamente en gran parte de la subregión la deforestación, la ganadería extensiva, la intensidad agrícola y la urbanización han modificado la mayoría de las zonas boscosas, principalmente en Ovejas y San Onofre. Teniendo en cuenta esta problemática fueron creadas 2 reservas naturales protectoras: Reserva forestal protectora Serranía de Coraza y Montes de María, departamento de Sucre, y el Santuario de fauna y flora Los Colorados, departamento de Bolívar (Aguilera, 2013).

Para los muestreos se realizaron visitas previas en diferentes localidades de los Montes de María con el fin de ubicar áreas con amplias coberturas de vegetación, sombra, incidencia de luz y la disponibilidad de agua durante todo el año; 


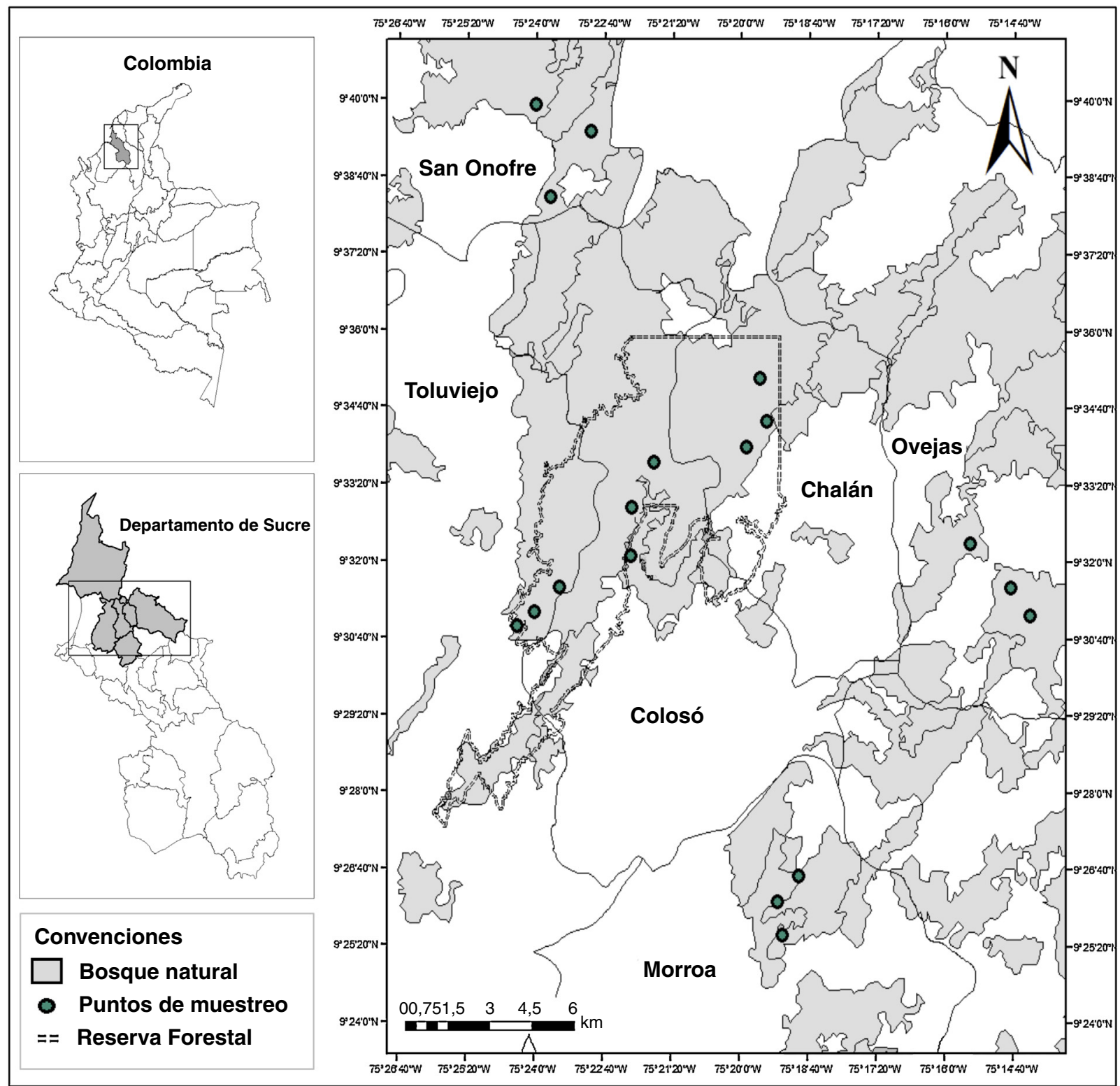

Figura 1. Ubicación geográfica del área de estudio; localidades de muestreo y posición de los sitios de recolecta en los Montes de María (Sucre-Colombia).

en otras palabras, sitios con las condiciones mínimas para el crecimiento de los briófitos (García et al., 2016). Se eligieron 6 sitios que se distribuyeron en los municipios de Colosó, Chalán, Toluviejo, Morroa, Ovejas y San Onofre (fig. 1), los cuales fueron denominados según su municipio. En estos se ubicaron al azar 3 transectos lineales de $100 \times 10 \mathrm{~m}$ y se recolectaron ejemplares de briófitos según las especificaciones dadas por García et al. (2016) y Orrego (2005); también se tomaron datos como la altitud, coordenadas geográficas y se realizó una estimación de la cobertura a través de una plantilla cuadriculada de acetato de $30 \times 20 \mathrm{~cm}^{2}$ (cuadros de $1 \times 1 \mathrm{~cm}$ ) modificada de Iwatsuki (1960).

Una vez finalizado el proceso de recolecta el material fue herborizado según los procedimientos convencionales para briófitos (García et al., 2016) y posteriormente identificados mediante las claves dicotómicas en Churchill y Linares (1995), Gradstein
(1991), Gradstein, Churchill y Allen (2001), Sharp, Crum y Eckeckeckel (1994) y Uribe y Aguirre (1997). Adicionalmente, se utilizó bibliografía especializada para Calymperaceae (Reese, 1993), Frullaniaceae (Gradstein y Uribe, 2011), Pottiaceae (Zander, 1993) y Lejeuneaceae (Gradstein, 1994); de igual forma, en los casos donde fue necesario, los ejemplares fueron corroborados por expertos. Finalmente, fueron consultadas bases de datos en línea como Bernal, Gradstein y Celis 2016, Boyle et al. (2013) y Missouri Botanical Garden (http://www.tropicos.org/) para actualizar la nomenclatura de las especies.

El material identificado fue tabulado por áreas de muestreo y en función de esta información se realizaron estimaciones de diversidad $\alpha$ y $\beta$. La diversidad $\alpha$ fue calculada según lo establecido por Jost (2006) en: ${ }^{0} \mathrm{D},{ }^{1} \mathrm{D}$ y ${ }^{2} \mathrm{D}$. En el caso de la de $\beta$ esta fue estimada según el reemplazo de especies mediante el índice 
de Whittaker (Moreno, 2001) y la similitud por distancia a través de una matriz entre localidades de muestreo, a la cual fue aplicado el coeficiente de similitud Bray-Curtis, y sobre éste se realizó un análisis clúster para agrupar las áreas de muestreo por su composición florística. La matriz de similitud se construyó a partir de los datos de abundancia relativa, con previo ajuste a raíz cuarta para disminuir el efecto de las especies dominantes (comunes) en comparación con las especies raras (Field, Clarke y Warwick, 1982). Los cálculos para diversidad $\alpha$ se realizaron con el programa PRIMER 6.1.7 (Clarke y Gorley, 2006) y para $\beta$ con PAST (Hammer, Harper y Ryan, 2001).

Para estimar la $\Delta^{+}$y $\Lambda^{+}$se usaron los sistemas de clasificación para musgos y hepáticas de Goffinet y Shaw (2009) y Söderström et al., (2015), con lo cual se estableció la jerarquía taxonómica para cada especie; posteriormente esta información fue analizada por medio la función TAXDTDEST del software PRIMER 6.1.7 (Clarke y Gorley, 2006). Para esta investigación las localidades con valores altos de $\Delta^{+}$y con menor $\Lambda+$ son las de mayor diversidad taxonómica (Clarke y Warwick, 2001).

\section{Resultados}

Se registraron 63 especies, 42 géneros y 23 familias (tabla 1). Los musgos estuvieron mejor representados con 38 especies, 29 géneros y 18 familias. Las hepáticas presentaron 25 especies, 13 géneros y 5 familias; de los taxones identificados en este estudio 21 son nuevos registros para el departamento de Sucre y 9 para el Caribe colombiano (tabla 1 ).

Con 17 especies Lejeuneaceae fue la familia más rica, seguida por Sematophyllaceae (6 especies), Fissidentaceae y Pottiaceae (con 5 cada una). Los géneros sobresalientes fueron: Lejeunea (8 especies), a este le siguieron Fissidens (6) Plagiochila y Sematophyllum (3 cada uno); los demás presentaron de una a 2 especies. En relación con la especie las más comunes y dominantes fueron Calymperes erosum y Hyophila involuta, mientras que 12 especies fueron raras con un solo registro y exclusivas de una localidad (tabla 1).

El análisis de diversidad $\alpha$ reveló que las localidades son significativamente diferentes en el número de especies $\left({ }^{0} \mathrm{D}\right)$. Colosó y Morroa presentaron los valores más altos de riqueza de especies, siendo Colosó 1.16 veces más diverso que Toluviejo y 1.5 veces más que Ovejas al comparar los valores de ${ }^{1} \mathrm{D}$. En cuanto a Chalán y San Onofre la diversidad disminuyó en $\sim 61 \%$ en comparación con Colosó. Esta misma tendencia se observó en cuanto a ${ }^{2} \mathrm{D}$, dado que Colosó y Morroa registraron el mayor número de especies dominantes, y San Onofre es la localidad con menor dominancia (fig. 2).

Con respecto a los resultados de diversidad $\beta$, en el análisis de clasificación se distinguieron grupos de localidades con un nivel de similitud mayor al 55\% (fig. 3): el grupo 1 conformado por Morroa-Ovejas y el grupo 2 integrado por Colosó-Toluviejo, mientras que San Onofre y Chalán se muestran independientes a estas agrupaciones. Por su parte, al aplicar el índice de Whittaker se obtuvo un total de 1.185 comunidades virtuales de las 6 posibles en el sitio de muestreo. En un análisis pareado entre localidades para establecer el porcentaje de recambio de especies necesario para conformar una sola comunidad, los resultados
Tabla 1

Listado de especies y su distribución en el área de estudio.

\begin{tabular}{|c|c|c|}
\hline Familias & Especies & Localidades \\
\hline \multicolumn{3}{|l|}{ Musgos } \\
\hline \multirow[t]{2}{*}{ Bryaceae } & $\begin{array}{l}\text { Anomobryum conicum } \\
\text { (Hornsch.) Broth. }\end{array}$ & $\mathrm{Tv}$ \\
\hline & $\begin{array}{l}\text { Mielichhoferia megalocarpum } \\
\text { (Arn.) Mitt. }\end{array}$ & $\mathrm{Cl}, \mathrm{Ch}, \mathrm{Tv}$ \\
\hline Bartramiaceae & $\begin{array}{l}\text { Philonotis uncinata (Schwägr.) } \\
\text { Brid. }\end{array}$ & $\mathrm{Mr}, \mathrm{Ov}$ \\
\hline Calymperaceae & Calymperes erosum Müll. Hal. & $\begin{array}{l}\mathrm{Cl}, \mathrm{Ch}, \mathrm{Tv}, \mathrm{Mr} \\
\text { Ov, So }\end{array}$ \\
\hline Hookeriaceae & $\begin{array}{l}\text { Hookeria acutifolia Hook. y } \\
\text { Grev. }\end{array}$ & $\begin{array}{l}\mathrm{Cl}, \mathrm{Ch}, \mathrm{Tv}, \mathrm{Mr}, \\
\text { Ov, So }\end{array}$ \\
\hline Fabroniaceae & Fabronia ciliaris (Brid.) Brid. & $\mathrm{Cl}, \mathrm{Tv}, \mathrm{Mr}, \mathrm{Ov}$ \\
\hline \multirow[t]{3}{*}{ Fissidentaceae } & $\begin{array}{l}\text { Fissidens prionodes Mont. } \\
\text { Fissidens flaccidus Mitt. } \\
\text { Fissidens dissitifolius Sull. }\end{array}$ & $\begin{array}{l}\mathrm{Cl}, \mathrm{Ch}, \mathrm{Tv}, \mathrm{Mr}, \mathrm{Ov} \\
\mathrm{Ch}, \mathrm{Tv}, \mathrm{So} \\
\mathrm{Cl}, \mathrm{Ch}, \mathrm{Tv}, \mathrm{Mr} \\
\text { Ov, So }\end{array}$ \\
\hline & Fissidens steerei Grout & $\mathrm{Cl}$ \\
\hline & Fissidens pellucidus Hornsch. & $\mathrm{Ch}, \mathrm{Tv}, \mathrm{Mr}, \mathrm{Ov}$ \\
\hline Hypnaceae & $\begin{array}{l}\text { Chryso-hypnum diminutivum } \\
\text { (Hampe) W.R.Buck* }\end{array}$ & $\mathrm{Cl}, \mathrm{Tv}, \mathrm{Mr}, \mathrm{So}$ \\
\hline Leucomiaceae & $\begin{array}{l}\text { Leucomium strumosum } \\
\text { (Hornsch.) Mitt. }\end{array}$ & $\mathrm{Cl}$ \\
\hline Meteoriaceae & $\begin{array}{l}\text { Meteorium nigrescens (Hedw.) } \\
\text { Dozy \& Molk.* }\end{array}$ & Tv, Ov, So \\
\hline \multirow[t]{2}{*}{ Thuidiaceae } & Thuidium tomentosum Schimp. & $\mathrm{Cl}, \mathrm{Ch}, \mathrm{Mr}$, So \\
\hline & $\begin{array}{l}\text { Pelekium involvens (Hedw.) A. } \\
\text { Touw }^{\mathrm{a}}\end{array}$ & $\mathrm{Cl}, \mathrm{Ch}, \mathrm{Tv}, \mathrm{Mr}, \mathrm{Ov}$ \\
\hline \multirow[t]{5}{*}{ Pottiaceae } & Tortella alpicola Dixon $^{\mathrm{a}, \mathrm{b}}$ & $\mathrm{Cl}, \mathrm{Tv}$ \\
\hline & Didymodon sp. & $\mathrm{Tv}, \mathrm{Mr}$ \\
\hline & $\begin{array}{l}\text { Dolotortula mniifolia (Sull.) R.H. } \\
\text { Zander }{ }^{\mathrm{a}, \mathrm{b}}\end{array}$ & Ov, So \\
\hline & $\begin{array}{l}\text { Hyophila involuta (Hook.) A. } \\
\text { Jaeger }\end{array}$ & $\begin{array}{l}\mathrm{Cl}, \mathrm{Tv}, \mathrm{Mr}, \mathrm{Ch} \\
\mathrm{Ov}, \mathrm{So}\end{array}$ \\
\hline & $\begin{array}{l}\text { Hyophiladelphus agrarius } \\
\text { (Hedwig) R. H. Zander }\end{array}$ & $\mathrm{Cl}$ \\
\hline \multirow[t]{6}{*}{ Sematophyllaceae } & $\begin{array}{l}\text { Trichosteleum papillosum } \\
\text { (Hornsch.) A. Jaeger } r^{\mathrm{a}, \mathrm{b}}\end{array}$ & $\mathrm{Mr}$ \\
\hline & Trichosteleum fluviale & $\mathrm{Cl}, \mathrm{Mr}$ \\
\hline & $\begin{array}{l}\text { Sematophyllum subsimplex } \\
\text { (Hedw.) Mitt. }{ }^{\mathrm{a}}\end{array}$ & $\mathrm{Cl}, \mathrm{Mr}$ \\
\hline & $\begin{array}{l}\text { Sematophyllum swartzii } \\
\text { (Schwägr.) W.H. Welch y H.A. } \\
\text { Crum }^{\mathrm{a}}\end{array}$ & $\mathrm{Mr}$ \\
\hline & $\begin{array}{l}\text { Sematophyllum subpinnatum } \\
\text { (Brid.) E. Britton }{ }^{\mathrm{a}}\end{array}$ & $\mathrm{Ch}$ \\
\hline & Taxithelium planum (Brid.) Mitt.* & $\mathrm{Ch}$, So \\
\hline Stereophyllaceae & $\begin{array}{l}\text { Eulacophyllum cultelliforme } \\
\text { (Sull.) W.R. Buck y R.R. Irelan }\end{array}$ & $\mathrm{Cl}, \mathrm{Tv}, \mathrm{Mr}, \mathrm{Ov}$ \\
\hline \multirow[t]{4}{*}{ Neckeraceae } & $\begin{array}{l}\text { Neckeropsis undulata (Hedw.) } \\
\text { Reichardt }\end{array}$ & $\mathrm{Cl}, \mathrm{Tv}, \mathrm{Ov}, \mathrm{So}$ \\
\hline & $\begin{array}{l}\text { Neckeropsis disticha (Hedw.) } \\
\text { Kindb. }^{\text {a }}\end{array}$ & $\mathrm{Cl}, \mathrm{Ch}$, So \\
\hline & $\begin{array}{l}\text { Porotrichum substriatum } \\
\text { (Hampe) Mitt. }\end{array}$ & $\mathrm{Cl}, \mathrm{Tv}, \mathrm{Ov}, \mathrm{So}$ \\
\hline & Porotrichodendron lindigi ${ }^{\mathrm{a}}$ & $\mathrm{Cl}, \mathrm{Ch}, \mathrm{Tv}, \mathrm{Mr}, \mathrm{Ov}$ \\
\hline Octoblepharaceae & Octoblepharum albidum $\mathrm{Hedw}^{\mathrm{a}}$ & $\mathrm{Cl}, \mathrm{Tv}, \mathrm{Mr}, \mathrm{Ov}$ \\
\hline \multirow[t]{2}{*}{ Pilotrichaceae } & $\begin{array}{l}\text { Callicostella pallida (Hornsch.) } \\
\text { Ångström }^{\text {a }}\end{array}$ & $\mathrm{Cl}, \mathrm{Ch}, \mathrm{Tv}$ \\
\hline & $\begin{array}{l}\text { Cyclodictyon albicans (Hedw.) } \\
\text { Kuntze }\end{array}$ & $\mathrm{Cl}, \mathrm{Ch}$ \\
\hline Rutenbergiaceae & $\begin{array}{l}\text { Pseudocryphaea domingensis } \\
\text { (Spreng.) W.R. Buck }\end{array}$ & $\mathrm{Ch}, \mathrm{Mr}, \mathrm{Ov}, \mathrm{So}$ \\
\hline Splacnobryaceae & $\begin{array}{l}\text { Splachnobryum obtusum (Brid.) } \\
\text { Müll. Hal. }\end{array}$ & $\mathrm{Cl}, \mathrm{Tv}, \mathrm{Mr}$, So \\
\hline
\end{tabular}


Tabla 1 (continuación)

\begin{tabular}{|c|c|c|}
\hline Familias & Especies & Localidades \\
\hline \multicolumn{3}{|l|}{ Hepáticas } \\
\hline \multirow{3}{*}{ Frullaniaceae } & Frullania cuencensis Taylor ${ }^{\mathrm{a}}$ & $\mathrm{Cl}, \mathrm{Mr}, \mathrm{Ov}$, So \\
\hline & Frullania riojaneirensis (Raddi) & $\mathrm{Cl}, \mathrm{Ch}, \mathrm{So}$ \\
\hline & Spruce $^{\mathrm{a}}$ & \\
\hline \multirow{18}{*}{ Lejeuneaceae } & Archilejeunea conf. badia & So \\
\hline & Blepharolejeunea conf. & So \\
\hline & Incongrua $a^{\mathrm{a}}$ & \\
\hline & Bryopteris diffusa (Sw.) Nees ${ }^{\mathrm{a}}$ & $\mathrm{Mr}$ \\
\hline & $\begin{array}{l}\text { Caudalejeunea lehmanniana } \\
\text { (Gottsche et al.) A. Evans } \mathrm{s}^{\mathrm{a}, \mathrm{b}}\end{array}$ & So \\
\hline & Ceratolejeunea conf. Confusa ${ }^{\mathrm{a}}$ & So \\
\hline & Cheilolejeunea sp. & $\mathrm{Ch}, \mathrm{Tv}, \mathrm{Mr}, \mathrm{Ov}$ \\
\hline & $\begin{array}{l}\text { Mastigolejeunea plicatiflora } \\
\text { (Spruce) Steph. }\end{array}$ & $\mathrm{Cl}, \mathrm{Ch}, \mathrm{Mr}$ \\
\hline & $\begin{array}{l}\text { Mastigolejeunea auriculata } \\
\text { (Wilson y Hook.) Schiffner }\end{array}$ & $\mathrm{Cl}$ \\
\hline & Microlejeunea sp. & $\mathrm{Cl}, \mathrm{Ch}$ \\
\hline & Lejeunea deplanata Nees & $\mathrm{Cl}, \mathrm{Tv}$ \\
\hline & Lejeunea flava (Sw.) Nees & $\begin{array}{l}\mathrm{Cl}, \mathrm{Ch}, \mathrm{Tv}, \mathrm{Mr} \text {, } \\
\text { Ov, So }\end{array}$ \\
\hline & Lejeunea trinitensis Lindenb & $\mathrm{Cl}, \mathrm{Ch}, \mathrm{Tv}, \mathrm{So}$ \\
\hline & Lejeunea sp.1 & $\mathrm{Cl}$ \\
\hline & Lejeunea sp.2 & $\mathrm{Cl}, \mathrm{Ch}, \mathrm{Mr}$, So \\
\hline & Lejeunea sp.3 & $\mathrm{Cl}, \mathrm{Ch}, \mathrm{Tv}$, So \\
\hline & Lejeunea sp.4 & So \\
\hline & Lejeunea sp.5 & Ch, So \\
\hline \multirow{2}{*}{$\begin{array}{l}\text { Marchantia- } \\
\text { ceae }\end{array}$} & Marchantia chenopoda $\mathrm{L}$. & $\mathrm{Cl}$, So \\
\hline & $\begin{array}{l}\text { Marchantia inflexa Nees y } \\
\text { Mont. }^{\mathrm{a}, \mathrm{b}}\end{array}$ & $\mathrm{Cl}, \mathrm{Mr}$, So \\
\hline \multirow{3}{*}{$\begin{array}{l}\text { Plagiochila- } \\
\text { ceae }\end{array}$} & Plagiochila fuscolutea Taylor & $\mathrm{Cl}, \mathrm{Mr}, \mathrm{Ov}$ \\
\hline & Plagiochila sp.1 & $\mathrm{Cl}, \mathrm{Ch}, \mathrm{Tv}, \mathrm{Mr}, \mathrm{Ov}$ \\
\hline & Plagiochila sp.2 & $\mathrm{Ch}$ \\
\hline Targioniaceae & Targionia hypophylla $\mathrm{L}^{\mathrm{a}}$ & $\mathrm{Ov}$ \\
\hline
\end{tabular}

Ch: Chalán; Cl: Colosó; Mr: Morroa; Ov: Ovejas; So: San Onofre; Tv: Toluviejo.

${ }^{a}$ Nuevo registro para Sucre

${ }^{b}$ Nuevo registro para el Caribe colombiano.

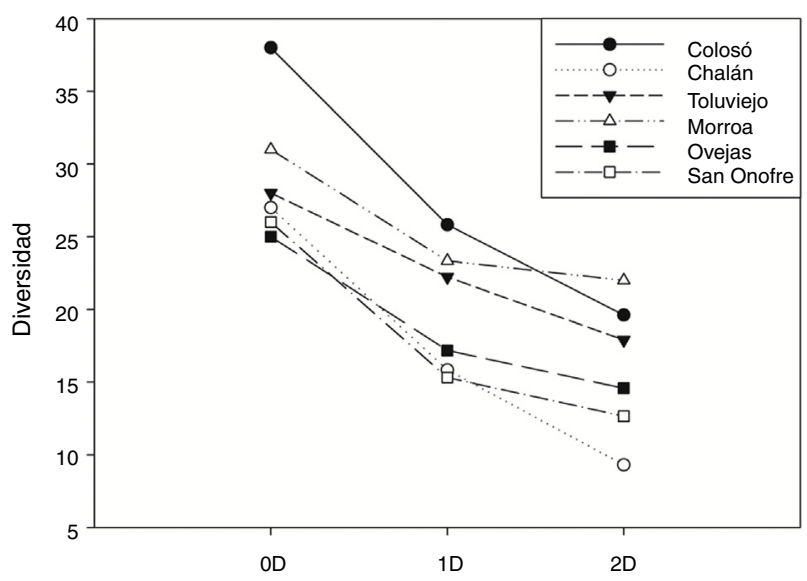

Figura 2. Diversidad alfa $\left({ }^{0} \mathrm{D},{ }^{1} \mathrm{D}\right.$ y $\left.{ }^{2} \mathrm{D}\right)$ de briófitos en los sitios de muestreos.

fueron similares a los observados en los análisis multivariados. Morroa y Ovejas solo requieren de un $33 \%$ de recambio para establecerse como una sola comunidad, es decir, tienen un porcentaje de $67 \%$ de similitud. En el caso de Colosó-Toluviejo presentan un $61 \%$ de similitud o un $39 \%$ de recambio de especies. Por el contrario, Chalán y San Onofre se muestran como

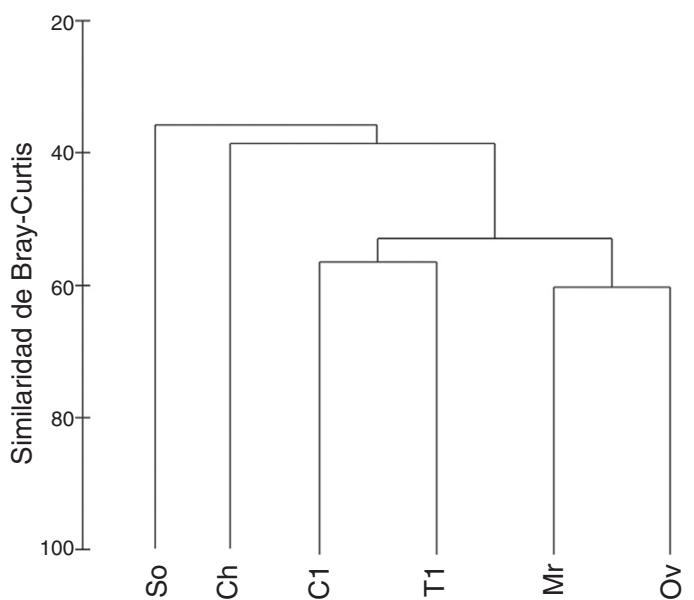

Figura 3. Dendrograma de clasificación (clúster) basado en la matriz de similitud de Bray-Curtis entre localidades de muestreo.

grupos independientes por mostrar el mayor porcentaje requerido para conformar una comunidad. San Onofre necesita entre 53-57\% de recambio de especies con todas las localidades para conformar al menos una comunidad, mientras que Chalán entre un $47 \%$ a $57 \%$.

En relación con los análisis de distinción taxonómica promedio, la gráfica de $\Delta^{+}$muestra que los valores se ubicaron dentro de los contornos probabilísticos de la distribución esperada (fig. 4A). Los valores de Colosó, Morroa y Ovejas se encontraron cercanos a la media estimada; mientras que los de Toluviejo, Chalán y San Onofre se ubicaron por debajo del límite inferior de confianza y distantes de la media esperada, a consecuencia de la baja complejidad taxonómica (fig. 4 A). Las localidades con mayor distinción taxonómica fueron Colosó y Morroa (tabla 2), siendo Colosó el de mayor cobertura taxonómica. Un resultado importante es que Ovejas, a pesar de ser una de las localidades con menor diversidad, obtuvo una mayor distinción taxonómica promedio en comparación con Toluviejo, Chalán y San Onofre (tabla 2).

En cuanto a la variación de la distinción taxonómica los datos se localizaron dentro de los contornos probabilísticos y por debajo de la media esperada, excepto Colosó, que se ubicó fuera del rango probabilístico, al presentar la menor variabilidad en su estructura taxonómica (fig. 4 B). El valor más alto fue alcanzado por San Onofre y Chalán quienes se ubicaron por encima del promedio esperado, a diferencia de las demás localidades (tabla 2).

\section{Discusión}

Los resultados muestran que las comunidades de briófitos no son homogéneas en su composición, abundancia y dominancia de taxones. Los grupos se caracterizaron por presentar pocas especies dominantes, con abundancias intermedias y un número particular de linajes raros. En otras palabras, ciertas condiciones ambientales al interior de cada punto o hábitat pueden favorecer o no a ciertas especies, dando lugar a comunidades delimitadas para cada localidad y taxones compartidos (Gimingham y Birse, 1957). 


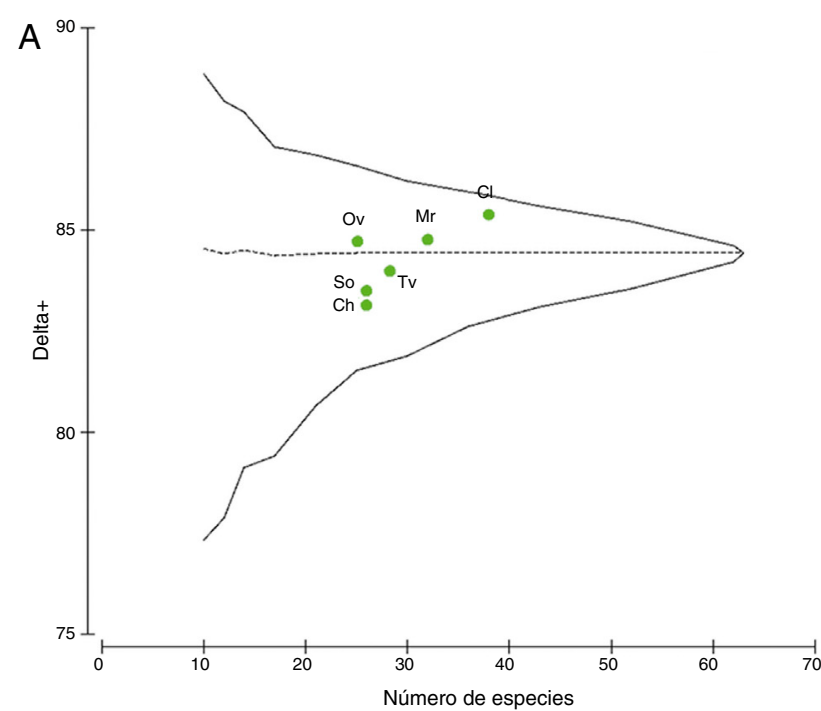

B

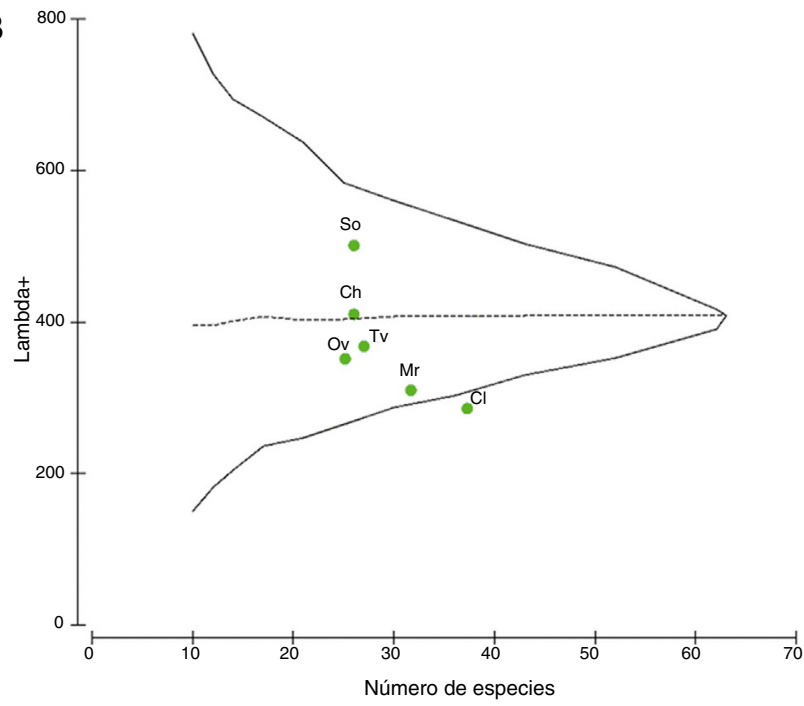

Figura 4. Distinción taxonómica promedio (A) y variación taxonómica (B) de briófitos en las localidades de muestreo. La línea horizontal segmentada representa la media esperada y la continua, los límites de confianza (95\%). Los puntos simbolizan los valores observados para cada sitio. Convenciones en la tabla 1.

El análisis de diversidad $\alpha$ reveló que Colosó y Morroa fueron más diversos en comparación con las otras localidades, lo cual puede ser explicado por los siguientes argumentos. Por un lado, están las diferencias locales con respecto a las variaciones ambientales como la hidrología, cobertura vegetal superior, suelo, agua y disponibilidad de luz. Por ejemplo, Colosó posee 7 microcuencas como resultado de la filtración de aguas subterráneas (Verbel, 2009), permitiendo que la oferta hídrica sea permanente sobre estas zonas, proporcionando un hábitat ideal para su colonización. Del mismo modo, estas características podrían estar compartidas con Morroa, ya que el recurso hídrico se mantiene a partir de los mantos acuíferos que existen en esta zona (Promontes, 2003). Empero, este factor no es constante para las demás zonas y su duración es muy corta, lo cual determina las especies que pueden sobrevivir a períodos de humedad largos o cortos. También, están las colinas y montañas (Serranía de Coraza), que junto a los bancos de nieblas (al amanecer y
Tabla 2

Distinción y variación taxonómica de briófitos en los sitios de muestreos.

\begin{tabular}{llll}
\hline Sitios de muestreo & $\mathrm{S}$ & $\Delta^{+}$ & $\Lambda^{+}$ \\
\hline Colosó & 38 & 85.38 & 234.93 \\
Chalán & 26 & 84.12 & 410.08 \\
Toluviejo & 27 & 83.42 & 339.55 \\
Morroa & 32 & 84.73 & 298.28 \\
Ovejas & 25 & 84.40 & 317.09 \\
San Onofre & 26 & 83.14 & 500.92 \\
\hline
\end{tabular}

$\Delta^{+}$: distinción taxonómica promedio; $\Lambda^{+}$: variación de la distinción taxonómica; $S$ : riqueza de especies.

atardecer) que provienen del golfo pueden incrementar la concentración de humedad (García et al., 2016), y por lo tanto la oferta de agua necesaria para el crecimiento de briófitos.

Otro factor que puede afectar la diversidad de estos grupos de plantas es la deforestación, tal como fue observada en Ovejas y San Onofre, áreas con valores de diversidad inferiores a Morroa y Colosó. La deforestación puede ocasionar varios procesos que afectan puntualmente a los briófitos, tales como reducción de la sombra, disminución de la humedad y la oferta de angiospermas que sirven como forófitos, elementos que en consideración a estas zonas Morroa y Colosó presentan sobre gran parte del año y por lo tanto un hábitat adecuado para el crecimiento de un mayor número de linajes diferentes.

En cuanto a diversidad $\beta$ el recambio promedio (1.185) entre las 6 localidades mostró poca variación, por lo tanto, la diferencia de especies entre una localidad a otra no es muy representativa; estos resultados son coherentes con el análisis clúster, considerando que la similitud fue mayor a $55 \%$, indicando bajo recambio de especies. Esta baja diversidad beta conlleva considerar que las variables ambientales tienden a ser homogéneas en el área de estudio y que podría existir una similitud ecológica entre microhábitats. En este sentido, las características ambientales similares entre las localidades parecen tener un rol importante en estos resultados; por ejemplo, la semejanza de Toluviejo-Colosó podría estar relacionada por la continuidad de sus bosques, ya que ambos pertenecen a la reserva forestal. En el caso de Morroa-Ovejas su similitud puede ser el reflejo de algún tipo de conexión histórica antes de la llegada del hombre al área de estudio.

Los análisis de $\Delta^{+}$y $\Lambda^{+}$indican que Colosó y Morroa son los sitios de mayor diversidad taxonómica, de manera que los ensamblajes están compuestos por un mayor número de géneros y familias que están menos relacionados taxonómicamente entre sí (Webb, Ackerly, McPeek y Donoghue, 2002). Es decir, que las variables abióticas como la precipitación y la humedad relativa, y las bióticas como mayor cobertura de la vegetación, han generado un ambiente apropiado para que coexistan un mayor número de especies que no están relacionadas taxonómicamente (Grass, Brandl, Botzat, Neuschulz y Farwig, 2015). Por otra parte, los valores de $\Delta^{+}$entre Chalán y Toluviejo fueron muy similares (tabla 2); sin embargo, en Chalán hubo mayor variación de la distinción taxonómica, lo que sugiere que en Toluviejo la mayoría de las especies pertenecen a linajes menos 
relacionados; de manera que, hacia Chalán, se incrementa la probabilidad de encontrar especies más emparentadas, del mismo género o familia, y con mayor homogeneidad taxonómica. Es también notable que Ovejas obtuvo mayor distinción y menor variación, por tanto, su estructura taxonómica es más diversa y sus especies están poco relacionadas taxonómicamente entre sí en comparación con otras localidades.

Bajo el contexto anterior, las localidades analizadas que presentaron altos valores de distinción taxonómica promedio tienen mayor número de especies pertenecientes a distintos taxones superiores. Por tanto, se podría sugerir que Colosó, Morroa y Ovejas son los sitios que aumentan la variabilidad y diversidad taxonómica de este grupo en el área de estudio.

En relación con San Onofre la baja diversidad taxonómica encontrada en esta localidad reflejó la presencia de condiciones ambientales adversas y la distribución similar de las especies en las categorías taxonómicas (poca amplitud taxonómica), ocasionada posiblemente por la intervención antrópica y el deterioro progresivo del ambiente. En este sentido Clarke y Warwick (1998) afirman que cuando un área ostenta alteraciones ambientales las especies se encuentran muy relacionadas, pertenecientes al mismo género o familia, y en consecuencia los hábitats poseen bajos valores de distinción. En general, los índices taxonómicos coincidieron con los índices ecológicos, ya que el comportamiento de la $\Delta^{+}$y $\Lambda^{+}$permiten sugerir que la diversidad taxonómica de briófitos se ve afectada tanto por condiciones del hábitat como por factores antropogénicos (González, Umaña, Álvarez, Stevenson y Swenson, 2014; Prinzing et al., 2008).

En otros términos, la heterogeneidad del hábitat actúa como un filtro ambiental que define estructuralmente los mecanismos de ensamblajes de estos taxones. Es decir, que las variaciones microambientales son las que determinan los patrones estructurales de las comunidades de briófitos en el área de estudio. Sin embargo, la deforestación y sus causas son factores que ocasionan fuertes alteraciones en la calidad del hábitat o condiciones ambientales que modifican los grupos de briófitos. En tal sentido, se considera que las condiciones de perturbación y variaciones microambientales en las que se encuentran los fragmentos de bosque en la subregión Montes de María son determinantes en la estructuración de los grupos de estos taxones.

Montes de María, tal como ha sido descrito por Pizano y García (2014), es una zona altamente conservada, lo cual ha generado una gran diversidad de plantas, tal es el caso de los briófitos, organismos que inicialmente habían sido poco estimados en esta área; no obstante, con el trabajo de García et al. (2016) y los nuevos reportes dados en este estudio, además de incrementar más del $50 \%$ con respecto a lo reportado por García, también reporta varias especies como registros nuevos para Colombia y el Caribe.

Según lo anterior, es importante entender que otras zonas de bosque seco en el Caribe colombiano también pueden tener una alta riqueza de estos organismos poco estudiados en estos bosques, dado que se asumía que las condiciones de estos bosques no generaban las bases para su crecimiento.

\section{Agradecimientos}

Al proyecto "Análisis florístico y fitogeográfico sobre relictos de bosque seco tropical en el departamento de Sucre (Colombia)", financiado por la División de Investigaciones de la Universidad de Sucre. A Edgar Linares y al Instituto de Ciencias Naturales de la Universidad Nacional de Colombia por el soporte recibido durante el desarrollo de esta investigación.

\section{Referencias}

Aguilera, M. (2005). La economía del departamento de Sucre: ganadería y sector público. En Documentos de trabajo sobre economía regional. Bogotá: Banco de la República.

Aguilera, M. (2013). Montes de María: una subregión de economía campesina y empresarial. En Documentos de trabajo sobre economía regional. Bogotá: Banco de la República.

Aguirre, J. y Avendaño, K. (2008). Musgos en la región Caribe. En O. Rangel (Ed.), Colombia diversidad biótica VI: riqueza y diversidad de musgos y líquenes de Colombia (pp. 55-59). Bogotá: Instituto de Ciencias Naturales.

Aguirre, J. y Ruiz, C. (2001). Composición florística de la brioflora de la Serranía del Perijá (Cesar- Colombia): distribución y ecología. Caldasia, 23, 181-201

Alvarado, D. y Otero, J. (2015). Distribución espacial del bosque seco tropical en el Valle del Cauca, Colombia. Acta Biológica Colombiana, 20, 141-153.

Avendaño, K. y Aguirre, J. (2007). Los musgos (Bryophyta) de la región de Santa María-Boyacá (Colombia). Caldasia, 29, 59-71.

Avendaño, K. y Aguirre, J. (2009). La brioflora de la serranía de Perijá (CesarColombia). En O. Rangel (Ed.), Colombia diversidad biótica viII: media y baja montaña de la serranía del Perijá (pp. 189-221). Bogotá: Instituto de Ciencias Naturales.

Bernal, R., Gradstein, R. y Celis, M. (2016). Catálogo de plantas y líquenes de Colombia [consultado 2 Nov 2016]. Disponible en: http://catalogoplantasdecolombia.unal.edu.co/

Boyle, B., Enquist, B., Raygoza, J., Hopkins, N., Lu, Z., Matasci, N., et al. (2013). The taxonomic name resolution service: an online tool for automated standardization of plant names [consultado 30 Oct 2016]. Disponible en: http://tnrs.iplantcollaborative.org/how_cite.html.

Churchill, S. y Linares, E. (1995). Prodromus Bryologiae Novo-Granatensis. Introducción a la flora de musgos de Colombia. Bogotá: Biblioteca José Jerónimo Triana.

Clarke, K. y Gorley, R. (2006). Primer v6: user manual and tutorial. Plymouth, UK: Primer-E.

Clarke, K. y Warwick, R. (1998). A taxonomic distinctness index and its statistical properties. Journal of Applied Ecology, 35, 523-531.

Clarke, K. y Warwick, R. (2001). A further biodiversity index applicable to species lists: variation in taxonomic distinctness. Marine Ecology Progress Series, 216, 265-278.

Cuervo, A., Barbosa, C. y de la Ossa, J. (1986). Aspectos ecológicos y etológicos de primates con énfasis en Alouatta seniculus (Cebidae), de la región de Colosó, serranía de San Jacinto (Sucre), costa norte de Colombia. Caldasia, XIV, 68-70.

Field, J., Clarke, K. y Warwick, R. (1982). A practical strategy for analysing multispecies distributions patterns. Marine Ecology Progress Series, $8,37-52$.

Galván, S., Gómez, H., de la Ossa, J. y Fajardo, A. (2009). Biodiversidad en el área de influencia de la estación primates de Colosó, Sucre, Colombia. Revista Colombiana de Ciencia Animal, 1, 98-121.

García, S., Basilio, H., Herazo, F., Mercado, J. y Morales, M. (2016). Diversidad de briófitos en los Montes de María, Colosó (Sucre, Colombia). Colombia Forestal, 19, 41-52.

García, R. M., Moreno, C. E. y Bello, J. G. (2014). Renovando las medidas para evaluar la diversidad en comunidades ecológicas: el número de especies efectivas de murciélagos en el sureste de Tabasco, México. Therya, 2, 205-215. 
Gimingham, C. y Birse, E. (1957). Ecological studies on growth-form in bryophytes: I. Correlations between growth-form and habitat. Journal of Ecology, 45, 533-545.

Goffinet, B. y Shaw, A. (2009). Bryophyte biology. New York: Cambridge University Press.

González, S., Umaña, M., Álvarez, E., Stevenson, P. y Swenson, N. (2014). Phylogenetic alpha and beta diversity in tropical tree assemblages along regional-scale environmental gradients in northwest South America. Journal of Plant Ecology, 7, 145-153.

Gradstein, S. (1991). A key to the Colombian species of holotipous Lejeuneaceae (Hepaticae). Caldasia, 16, 429-438.

Gradstein, S. (1994). "Lejeuneaceae: Ptychantheae, Brachiolejeuneae". Flora Neotropica. New York: New York Botanical Garden Press.

Gradstein, S. R., Churchill, S. y Allen, N. (2001). Guide to the Bryophytes of Tropical America. New York: New York Botanical Garden Press.

Gradstein, R. y Uribe, J. (2011). A synopsis of the Frullaniaceae (Marchantiophyta) from Colombia. Caldasia, 33, 367-396.

Grass, I., Brandl, R., Botzat, A., Neuschulz, E. y Farwig, N. (2015). Contrasting taxonomic and phylogenetic diversity responses to forest modifications: comparisons of taxa and successive plant life stages in South African scarp forest. Plos One, 10, 1-20.

Halffter, G. (1992). La diversidad biológica de Iberoamérica I. Xalapa: Instituto de Ecología, A.C.

Hammer, Ø., Harper, D. y Ryan, P. (2001). Past: paleontological statistics software package for education and data analysis. Palaeontologia Electronica, $4,1-9$.

Herazo, V. F., Mercado-Gómez, J. D. y Mendoza, H. (2017). Estructura y composición florística del bosque seco tropical en los Montes de María (Sucre-Colombia). Ciencia en Desarrollo, 8, 79-90.

Holdridge, L. (1967). Life zone ecology. San José, Costa Rica: Tropical Science Center.

Iwatsuki, Z. (1960). The epiphytic bryophyte communities in Japan. Journal of the Hattori Botanical Laboratory, 22, 159-339.

Jost, L. (2006). Entropy and diversity. Oikos, 113, 363-375.

López, A. (2003). EOT: Esquema de ordenamiento territorial diagnóstico integral del territorio (2009). Municipio de Morroa, Departamento de Sucre: Alcaldía de Morroa.

Marulanda, L., Uribe, A., Velásquez, P., Montoya, M., Idárraga, A., López, M., et al. (2003). Estructura y composición de la vegetación de un fragmento de bosque seco en San Sebastián, Magdalena (Colombia). I. Composición de plantas vasculares. Actualidades Biológicas, 25, 17-30.

Moreno, C. (2001). Métodos para medir la biodiversidad. Zaragoza, España: Manuales y Tesis SEA Zaragoza.

Moreno, C., Barragán, F., Pineda, E. y Pavón, P. (2011). Reanalizando la diversidad alfa: alternativas para interpretar y comparar información sobre comunidades ecológicas. Revista Mexicana de Biodiversidad, 82, 1249-1261.
Orrego, O. (2005). Briófitos de Caldas: la Reserva de Planalto. Boletín CientíficoCentro de Museos-Museo de Historia Natural, 9, 31-50.

Pizano, C. y García, H. (2014). El bosque seco tropical en Colombia. Bogotá: Instituto de Investigación de Recursos Biológicos Alexander von Humboldt (IAVH).

Prinzing, A., Reiffers, R., Braakhekke, W., Hennekens, S., Tackenberg, O., Ozinga, W., et al. (2008). Less lineages-more trait variation: phylogenetically clustered plant communities are functionally more diverse. Ecology Letters, 11, 809-819.

Promontes. (2003). Programa de desarrollo y paz de los Montes de MaríaPromontes". Bogotá: PNUD, Corporación Territorios, Universidad de Cartagena, Cartagena.

Reese, W. (1993). "Calymperaceae". In Flora Neotropica. New York: New York Botanical Garden Press.

Rodríguez, G., Banda, K., Reyes, S. y Estupiñán, A. (2012). Lista comentada de las plantas vasculares de bosques secos prioritarios para la conservación en los departamentos de Atlántico y Bolívar (Caribe colombiano). Biota Colombiana, 13, 7-39.

Ruiz, C. y Aguirre, J. (2003). Las comunidades de briófitos y su relación con la estructura de la vegetación fanerogámica en el gradiente altitudinal de la serranía del Perijá (Cesar-Colombia). Tropical Bryology, 24, 101-113.

Santos, G. y Aguirre, J. (2010). Los musgos de la región de las Quinchas (Magdalena Medio, Colombia). Caldasia, 32, 257-273.

Sharp, A., Crum, H. y Eckeckeckel, P. (1994). The moss flora of México. Nueva York: New York Botanical Garden.

Söderström, L., Hagborg, A., Von Konrat, M., Bartholomew-Began, S., Bell, D., Briscoe, L., et al. (2015). World checklist of hornworts and liverworts. PhytoKeys, 59, 1-828.

Uribe, J. y Aguirre, J. (1997). Clave para los géneros de hepáticas de Colombia. Caldasia, 19, 13-27.

Van Reenen, G., Griffin, D. y Gradstein, S. (1984). Briófitos del transecto Buritacá-La Cumbre, Sierra Nevada de Santa Marta, Colombia. En T. Van der Hammen y P. Ruiz (Eds.), La Sierra Nevada de Santa Marta (Colombia), transecto Buritaca-La Cumbre. Estudios de ecosistemas tropandinos (pp. 177-183). Berlín: Cramer.

Verbel, C. (2009). EOT: esquema de ordenamiento territorial diagnóstico integral del territorio (2009). Municipio de Colosó, Departamento de Sucre. (Versión preliminar). Alcaldía de Colosó.

Warwick, R. y Clarke, R. (1995). New’biodiversity' measures reveal a decrease in taxonomic distinctness with increasing stress. Marine Ecology Progress Series, 129, 301-305

Webb, C., Ackerly, D., McPeek, M. y Donoghue, M. (2002). Phylogenies and community ecology. Annual Review of Ecology and Systematics, 33, 475-505.

Zander, R. (1993). Genera of the Pottiaceae: mosses of harsh environments. Búfalo, NY: Buffalo Society of Natural Sciences. 